Introduction: Children's motor coordination may affect their activity pattern and thereby influence their bodyfatness. The aim of the present study was to analyse the relationship between motor coordination and total body fat percentage, in a Portuguese sample of schoolchildren aged 9-12 years.

Method: The sample comprised 596 urban schoolchildren (girls 46.9\%), aged 9-12 years (mean 9.61 (SD 0.55) years) from North of Portugal. Total body fat percentage was calculated with Tetrapolar Bioelectrical Impedance Analysis, model Tanita TBF-300. Age- and sex-adjusted Z-scores computed total body fat percentage. Motor coordination levels were assessed with the Körperkoordination Test für Kinder (KTK) and children were classified according to age and sex KTK criteria (Schiling 1974). Date of birth, gender and school socio-economic status (eligible for benefit A, B or not eligible) were extracted from the school administrations records systems. School socio-economic status was used as a proxy measure of family socio-economic status.
Results: In motor coordination, $22 \cdot 4 \%$ girls showed disturbance of coordination; $37.7 \%$ insufficiencies of coordination; $39 \cdot 5 \%$ normal coordination and $0 \cdot 4 \%$ good coordination. Corresponding figures for boys were $7 \cdot 3 \%$; $36 \cdot 5 \% ; 54 \cdot 3 \%$ and $1 \cdot 9 \%$, respectively. Linear regression analysis showed that $Z$-scores total body fat percentage (unstandardized $B=-0 \cdot 258$, SE $0 \cdot 021, P<0 \cdot 001$ ) were negatively associated with motor coordination, after adjustment for socio-economic status.

Conclusions: Low motor coordination levels are negatively associated with total body fat percentage $Z$-scores. The early identification of children with poor motor coordination and/or high body fat percentage is crucial in order to implement and develop health-related behaviours.

Funding: Research relating to this abstract was funded by FCT-MCTES Grant (BD/43808/2008).

Reference: Schiling, F (1974) Korperkoordination Test fur Kinder, KTK. Beltz Test Gmbh, Weinheim.

\title{
44 - Relations between resting blood pressure (RBP), weight status and physical activity (PA) in British schoolchildren
}

\author{
Michael J Duncan ${ }^{1}$, Luke James ${ }^{2}$ and Layla Griffiths ${ }^{2}$ \\ ${ }^{1}$ Department of Biomolecular and Sports Science, Coventry University, UK: ${ }^{2}$ Department of Biology, Forensics and \\ Sport, University of Derby, UK
}

Introduction: The present study aimed to assess the relations between resting blood pressure (RBP), physical activity (PA) and weight status in a multiethnic sample of British children.

Method: 790, 10-14-year-old schoolchildren (444 boys, 346 girls) underwent assessment of RBP, height, body mass (from which BMI was calculated and classified according to International Obesity Task Force criteria) and completed the physical activity questionnaire for adolescents (PAQ-A) as a measure of PA following ethics approval and informed consent. Children were classified as being from 'white' ( $n$ 553), 'black' ( $n$ 51) and 'Asian' ( $n$ 186) backgrounds based on census classifications.

Results: Pearson's correlations indicated significant relations between BMI and systolic BP (SBP) $(r=0 \cdot 424$,
$P=0 \cdot 0001)$, diastolic BP (DBP) $(r=0 \cdot 224, P=0 \cdot 0001)$ and PA $(r=-0 \cdot 255, P=0 \cdot 0001)$. PA was significantly but weakly related to SBP $(r=-0 \cdot 103, P=0 \cdot 02)$ but not DBP $(P>0 \cdot 05)$. A series of 2 (gender) by 2 (weight status) by 3 (ethnic groups) ANCOVA controlling for age found that SBP was significantly higher in overweight/obese children compared with normal weight children $(P=0 \cdot 0001)$. This pattern was evident for $\operatorname{DBP}(P=0 \cdot 0001)$ and PA $(P=0 \cdot 001)$. PA score was higher in boys than girls $(P=0 \cdot 01)$. There were no ethnic differences in any variables (all $P>0 \cdot 05$ ).

Conclusions: RBP is higher and PA lower in overweight/obese children compared with normal weight children when controlling for age. Ethnic groups did not appear to influence RBP or PA in this sample.

\section{5 - Prevalence of cardiometabolic risk factors in overweight and obese adolescents}

\author{
K Anyfandakis ${ }^{1}$, G Dali ${ }^{1}$, E Kousta ${ }^{1}$, M Dolianiti $^{1}$, G Tsagaraki ${ }^{1}$, H Kallergis ${ }^{2}$, A Koulieri ${ }^{2}$, \\ A Tsitsika ${ }^{3,4}$ and A Papathanasiou ${ }^{1}$ \\ 'Department of Pediatric Endocrinology, Greece: ${ }^{2}$ Department of Dietetics, Greece: ${ }^{3}$ Aglaia Kyriakou Children's \\ Hospital, Athens, Greece: ${ }^{4}$ Adolescent Health Unit, 2nd Pediatric University Clinic, Greece
}


Introduction: Overweight and obesity is steadily increasing in Greek adolescents over the past years. The presence of risk factors for CVD in these children is very important for further preventive and therapeutic measures.

Aim: To investigate the prevalence of insulin resistance, hypertension dyslipidemia and hyperuricemia in overweight and obese adolescents.

Material and Method: A total of 327 adolescents (189 obese and 138 overweight - according to T Cole) (162 boys and 165 girls) aged 14 (SD 2) years (12-20) attending the obesity clinic were evaluated. Anthropometric data (height, weight), BP and laboratory investigations (fasting glucose, insulin, lipid profile, uric acid, OGTT) at presentation were included in the study.

Results: Elevated blood pressure (BP) was recorded in $32.7 \%$ of the obese and $15.3 \%$ of the overweight children $(P=0 \cdot 001) ; 25 \cdot 5 \%$ of the obese $v \cdot 12 \cdot 7 \%$ of the overweight had systolic $\mathrm{BP}>95$ th percentile $(P=0.008)$ and $20 \%$ v. $4.5 \%$ had diastolic $\mathrm{BP}>95 \%$ $(P<0.001) ; 10.9 \%$ of the obese $v .8 .4 \%$ of the overweight had elevated fasting glucose $(P=0 \cdot 474)$. The proportion of insulin resistance (HOMA-IR $>3 \cdot 16$ ) was significantly higher $(62 \cdot 2 \%)$ in obese compared with overweight children $(P=0.001) ; 7 \%$ of the obese $v .3 \%$ of the overweight had low HDL $(P=0 \cdot 12)$ and $12.6 \%$ of the obese $v .5 \cdot 2 \%$ of the overweight had triglycerides $>150 \mathrm{mg} /$ $\mathrm{dl}(P=0.009) ; 26 \cdot 3 \%$ of the obese $v .12 \%$ of the overweight had high uric acid $(P=0 \cdot 009)$.

Conclusions: The frequency of several cardiometabolic risk factors is significantly higher in obese compared with overweight Greek adolescents indicating the need for early intervention.

\title{
46 - Mild reductions in BMI Z-score through lifestyle modifica- tions seem to improve cardiovascular risk in a Portuguese pediatric population
}

\author{
H Nascimento ${ }^{1,2}$, S Rocha ${ }^{1,2}$, J Fernandes ${ }^{3}$, E Costa ${ }^{2,3}$, C Rego $^{4}$, HF Mansilha ${ }^{5}$, \\ P Rocha-Pereira ${ }^{6}$, A Quintanilha ${ }^{2,7}$, A Santos-Silva ${ }^{1,2}$ and L Belo ${ }^{1,2}$
}

'Departamento de Bioquimica, Faculdade de Farmacia, Universidade do Porto, Porto, Portugal: ${ }^{2}$ Instituto de Biologia Molecular e Celular (IBMC), Universidade do Porto, Porto, Portugal: ${ }^{3}$ Escola Superior de Biotecnologia, Universidade Catolica Portuguesa, Porto, Portugal: ${ }^{4}$ Unidade de Nutricao/Servico Pediatrico, UAG-MC, Hospital de S. Joao E.P.E., Faculdade de Medicina, Universidade do Porto, Porto, Portugal: ${ }^{5}$ Departamento Pediatrico do Hospital de Criancas D. Maria Pia, Porto, Portugal: ${ }^{6}$ Centro de Investigacao em Ciencias da Saude, Universidade da Beira Interior, Covilha, Portugal: ${ }^{7}$ Instituto de Ciencias Biomedicas Abel Salazar (ICBAS),

Universidade do Porto, Porto, Portugal

Introduction: Good lifestyle habits should be introduced early in an individual's life. We studied a natural progressive approach on changing obese children and adolescent's habits and how these changes might reflect in weight loss and cardiovascular risk markers.

Method: Obese paediatric patients from University Hospital S. Joao and of Children's Hospital Maria Pia, Porto, were asked to participate in a cross-sectional study: 148 obese children and adolescents (81 females (54.7\%); mean age: $11 \cdot 0$ years (4-16)) and thirty-three matching controls participated. Sixty obese patients also agreed to participate in a longitudinal study, with a second evaluation of cardiovascular risk markers one year after the lifestyle modification intervention. A substantial BMI reduction was defined as a decrease in BMI $Z$-score of 0.3 or more over the studied period (cut-off value).

Results: Cross-sectional study: compared with controls, obese patients presented with significantly higher homeostasis model assessment-insulin resistance (HOMA-IR), triglycerides, LDL-cholesterol, apo B, insulin and C-reactive protein concentrations, whereas their HDL-cholesterol and apo A levels were significantly lower. Longitudinal study: seventeen individuals (28.3\%) reached the BMI $Z$-score reduction cut-off value, which led to a significant reduction in triglycerides, cholesterol, LDL-cholesterol, apo B, glucose, insulin levels and HOMA-IR. The other forty-three children that did not reach the cut-off presented with a significant reduction in adiponectin values and increase in lipoprotein (a) concentration.

Conclusions: Small reductions in BMI Z-score may improve cardiovascular risk profile in obese children and adolescents, with particularly notorious effects in lipid profile and insulin sensitivity. Conversely, children bellow the cut-off presented with a worsened risk profile.

Funding: The present study was supported by a PhD grant (SFRH/ BD/61407/2009) attributed to Henrique Nascimento by Fundacao para a Ciencia e Tecnologia (FCT) - Portugal. 